\title{
A NOVEL APPROACH FOR EDGE DETECTION OF LOW CONTRAST SATELLITE IMAGES
}

\author{
K. K. Singh ${ }^{\mathrm{a},{ }^{*}}$, M. K. Bajpai ${ }^{\mathrm{a}}$, R. K. Pandey ${ }^{\mathrm{b}}$ \\ ${ }^{a}$ Computer Science \& Engineering, Indian Institute of Information Technology, Design \&Manufacturing Jabalpur, M.P., 482005 \\ India - (koushlandras, mkbajpai)@iiitdmj.ac.in \\ ${ }^{b}$ Department of Mathematical Sciences, Indian Institute of Technology (BHU) Varanasi, U.P., 221005, India- \\ rkpandey.mat@iitbhu.ac.in
}

\begin{abstract}
KEY WORDS: Sobel Edge Detector, Fractional Order Differentiator, Chebyshev Polynomial based Approximation, Satellite Images.
\end{abstract}

\begin{abstract}
:
Discrimination between texture edges and geometrical edges is very difficult in low contrast images. Satellite images are low contrast images. It is important to extract the edges that are not clearly visible in case of Satellite images. The present work encompasses a new edge detection algorithm using newly constructed differentiator. Chebyshev polynomial based fractional order differentiator has been used for filtering operation on an image. High pass and Low pass filters are designed with the concept of Quadrature Mirror Filter $(Q M F)$. Pre-processing has been performed by using this filter. Sobel edge detection method has been applied on this pre-processed image. The algorithm has been tested with two different satellite images.
\end{abstract}

\section{INTRODUCTION}

Satellite images have texture and smoothness as a dominating features present in them. Smoothness properties of satellite images drive us to apply the low pass filtering operation that will extract the texture edges present in the images. Edge detection has been extensively analysed in computer vision and image processing field from last three decades but it has not yet been entirely solved. There is no universal detection algorithm present in literature which can detect all scales of edges, irrespective of their shape, image type etc. Edge detection methods are image dependent. The purpose of edge detection approach is to find the discontinuities present in gray scale value in an image. Rosenfield et al. have introduced edge detection algorithm first time in 1971 (Rosenfield, 1971). Multiscaling edge detection methods have been proposed in literature (Man, 1982), (Marrand, 1980), (Wotkin, 1983). A large set of filters exists in image processing. The detection of edges, corners and other two dimensional features have been obtained by convolving the original image with directional filters having different orientation, size and shape. Mathematical morphology, Markav fields, Derivatives based approach with linear filtering etc. are major approach for edge detection. Canny edge detection algorithm is an optimal edge detection based on derivatives of the Gaussian (Canny, 1986). The other popular algorithms for edge detections are Roberts method, Sobel method, Prewitt operators, Finite impulse response filters with large kernel such as Canny filter, First order recursive filters, Shen filter, Second order recursive filter, Deriche filter, first order derivative of Gaussian function etc. (Yuille, 1986), (Prewitt, 1970), (Canny J, 1986), (Deriche, 1990), (Shen J, 1992). Deriche et.al. has proposed a new algorithm that is more efficient in noise filtering as well as edge detection (Deriche, 1990).

Wavelet transform has been applied with canny edge detector

*Corresponding author by Jie Hou et al (Jie, 2007). The other edge detection approaches that are popular among the researchers are differentiation based edge detection using logarithmic image processing (LIP) models , contrast-based methods, relaxation labeling techniques and anisotropic diffusion (Deng, 1998), (Johnson, 1990), (Iyengar, 1995), (Perona P, 1990).

Design of a fractional order differentiator based filter (FDD) is described in literature (Koushlendra, 2014). They have used Chebyshev polynomials for approximation of the filter function.

The present work encompasses design and development of an algorithm which uses a pre-processed image in Sobel edge detection algorithm. This pre-processing has been performed by using fractional order differentiator.

The structure of this paper is as follows. Section 2 discusses design of Chebyshev polynomial based fractional order filter. Section 3 describes newly designed algorithm for edge detection in low contrast images. Section 4 presents the details of experiments. Section 5 reports the results obtained from experiments. Section 6 describes the conclusions.

\section{DESIGN OF FILTER}

The detailed algorithm for design of fractional order differentiator is present in literature (Koushlendra, 2014). We are reproducing it here for better understanding.

Consider two higher order differentiable functions in $\mathbb{R}$ as $Y(t)$ and $\tilde{Y}(t)$ which are observed function and original function respectively. The observed function can be written as

$$
Y(t)=\tilde{Y}(t)+\xi(t)
$$

here $\xi(t)$ is error. The present work encompasses smoothing of observed function by the use of $n^{\text {th }}$ order derivative, $L$ point filtering window and $n$-degree polynomial approximation. 
Any function $Y(t)$ can be obtained by polynomial expansion expressed as follows (Chen D, 2011):

$Y(t)=\sum_{k=0}^{n} c_{k} T_{k}(t)$

$t=1,2,3 \ldots, L$ is the position of the $t^{\text {th }}$ point in the filtering window and $c_{k}$ is the $k^{\text {th }}$ coefficient of polynomial function. Least-square method is used for the estimate the coefficients $c_{k}$. Equation (2) can expanded in the following form

$$
\begin{aligned}
& T_{0}(1) c_{0}+T_{1}(1) c_{1}+T_{2}(1) c_{2}+\ldots+T_{n}(1) c_{n}=y_{1} \\
& T_{0}(2) c_{0}+T_{1}(2) c_{1}+T_{2}(2) c_{2}+\ldots+T_{n}(2) c_{n}=y_{2} \\
& T_{0}(3) c_{0}+T_{1}(3) c_{1}+T_{2}(3) c_{2}+\ldots+T_{n}(3) c_{n}=y_{3} \\
& \begin{array}{lllll}
\ldots & \ldots & \ldots & \ldots & \ldots
\end{array} \\
& \begin{array}{lllll} 
& \ldots & \ldots & \ldots & \ldots
\end{array} \\
& T_{0}(L) c_{0}+T_{1}(L) c_{1}+T_{2}(L) c_{2}+\ldots+T_{n}(L) c_{n}=y_{L}
\end{aligned}
$$

here $Y=\left[y_{1}, y_{2}, \ldots, y_{L}\right]^{T}$ denotes the measured function points in the filtering window. $T$ is a matrix of order $L \times(n+1)$ and can be defined as

$$
T=\left[\begin{array}{lllll}
T_{0}(1) & T_{1}(1) & T_{2}(1) & \cdots & T_{n}(1) \\
T_{0}(2) & T_{1}(2) & T_{2}(2) & \cdots & T_{n}(2) \\
\vdots & \vdots & \vdots & & \vdots \\
T_{0}(L) & T_{1}(L) & T_{2}(L) & \cdots & T_{n}(L)
\end{array}\right]
$$

The elements of matrix $\mathrm{T}$ are calculated by using chebyshev polynomial (Pandey R, 2014)

$$
T_{n+1}(t)=2 t T_{n}(t)-T_{n-1}(t)
$$

here $T_{0}(t)=1, T_{1}(t)=t$.

The vectors $\mathrm{C}$ storing the coefficients of the polynomial are obtained by following expression.

$$
C=\left(T^{T} T\right)^{-1} T^{T} Y
$$

Equation (5) and (6) are used to solve equation (3). It will result

$$
\hat{Y}=T C=T\left(T^{T} T\right)^{-1} T^{T} Y=W Y
$$

here $W$ denotes window's coefficient matrix. Smoothing can be performed by use of different window coefficient matrix.

Riemann-Liouville fractional order derivative can be expressed as

$$
{ }_{0} D_{x}^{\alpha} Y(t)=\frac{1}{\Gamma(l-\alpha)} \frac{d^{l}}{d t^{l}} \int_{0}^{x}(t-x)^{l-\alpha-1} f(x) d x
$$

here $0 \leq l-1<\alpha<l$, and $\Gamma(l-\alpha)$ is the Gamma function of $(l-\alpha) . \alpha$ is the positive order of differentiation and its value lies between $l-1$ to $l$.

The fractional order differentiator, corresponding to window coefficient matrix $W$, can be obtained by equation (8). Different properties of fractional order differentiation are applied on equation (7) and we will get (Oldham, K.B, 1974) and (Podulubny, I, 1998).

$\hat{Y}_{t}^{\alpha}=T_{t}^{\alpha} C=W_{t}^{\alpha} Y=c\left(T^{T} T\right)^{-1} T^{T} Y$
It is generalized form. Here $\hat{Y}_{t}^{\alpha}$ denotes the $\alpha^{\text {th }}$ derivative of the $t^{\text {th }}$ point in the filtering window, $W_{t}^{\alpha}$ denotes the $\alpha^{\text {th }}$ derivative coefficient vector of the $t^{\text {th }}$ point in the filtering window.

\section{Algorithm 1}

The algorithm can be expressed as follow

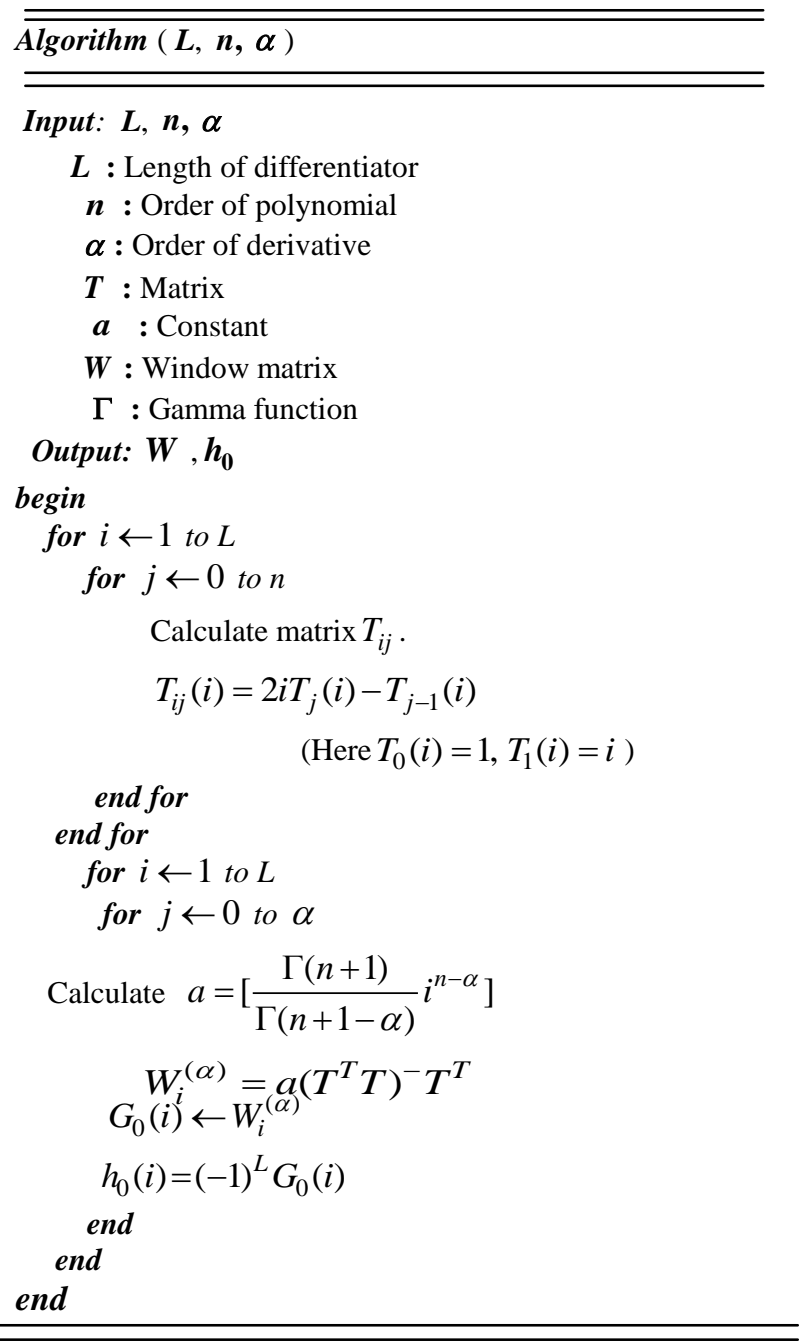

\section{ALGORITHM FOR EDGE DETECTION IN SATELLITE IMAGES}

Flow chart of the proposed algorithm has been shown in figure 1. The input image, $I$, has been normalized. The normalized image, $I_{n}$, has been processed with newly designed Chebyshev polynomial based fractional order filter. The low pass filtering operation and high pass filtering operation has been performed on normalized image.

Image, $I_{l r}$, is the output image of low pass filtering operation followed by relaxation coefficient. The result of low pass filtering operation has been processed with relaxation coefficient. The relaxation coefficient varies from 0 to 2 . Multiplication operation has been performed between output image of high pas filtering operation and $I_{l r}$. The Sobel edge detection algorithm has been applied on this pre-processed image for edge detection. 


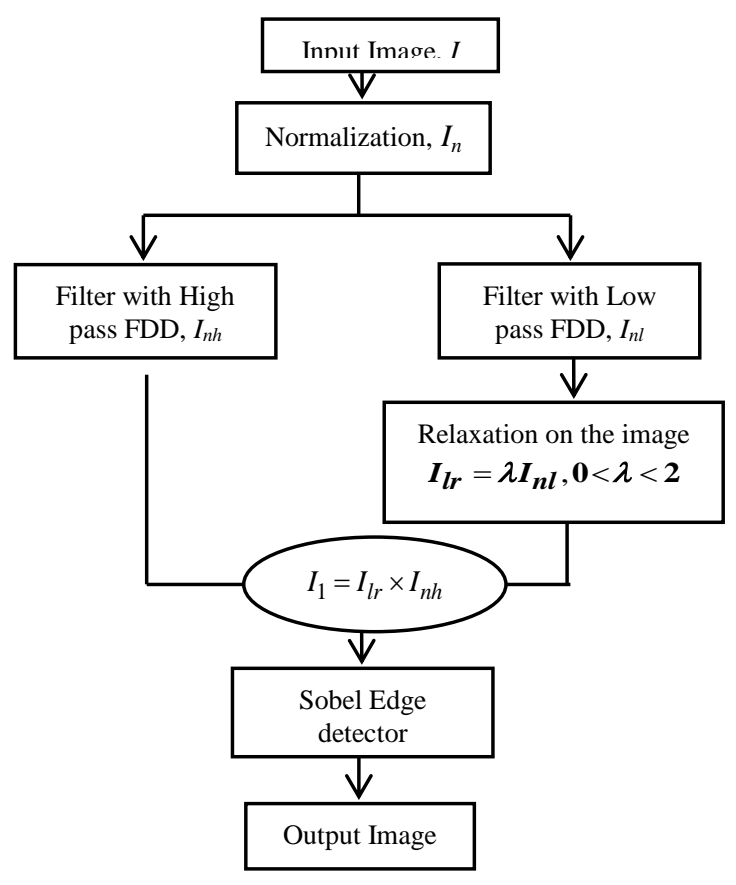

Figure 1. Flow chart of algorithm

\section{Algorithm 2}

Proposed algorithm is as follow

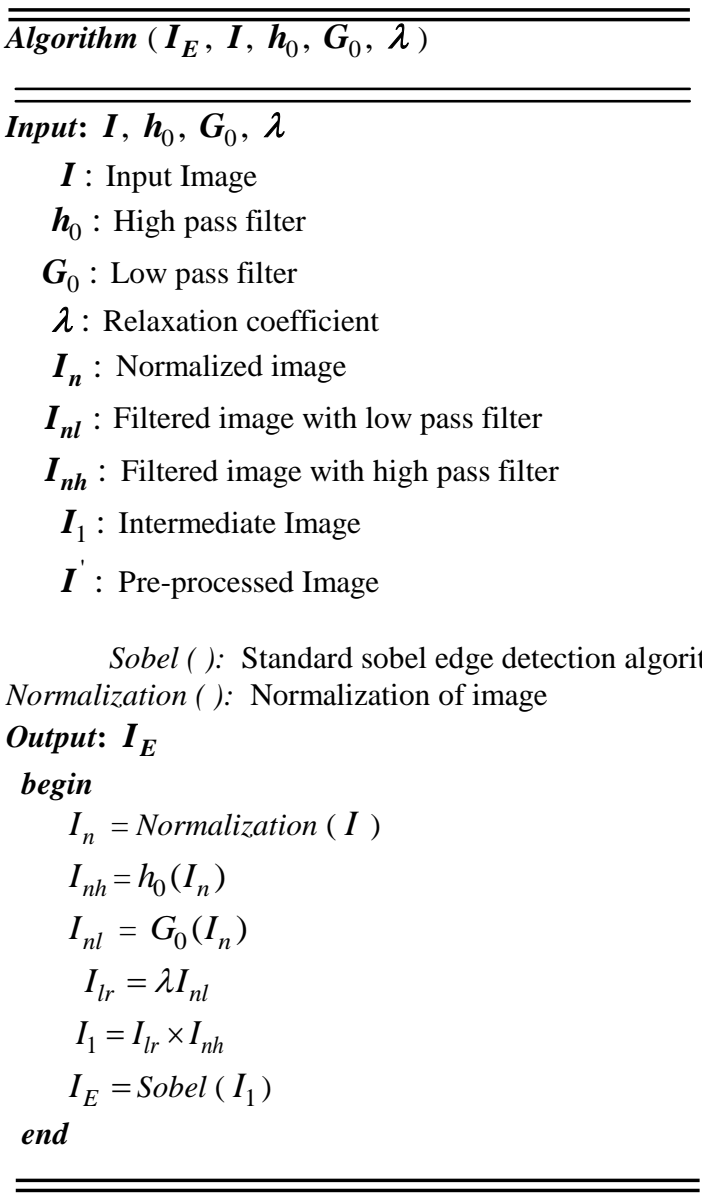

\section{EXPERIMENTS}

The proposed algorithm has been tested with two test images. Both satellite images are easily available in open domain (Photojournal, 2007). The images have smoothness properties as well as low contrast.
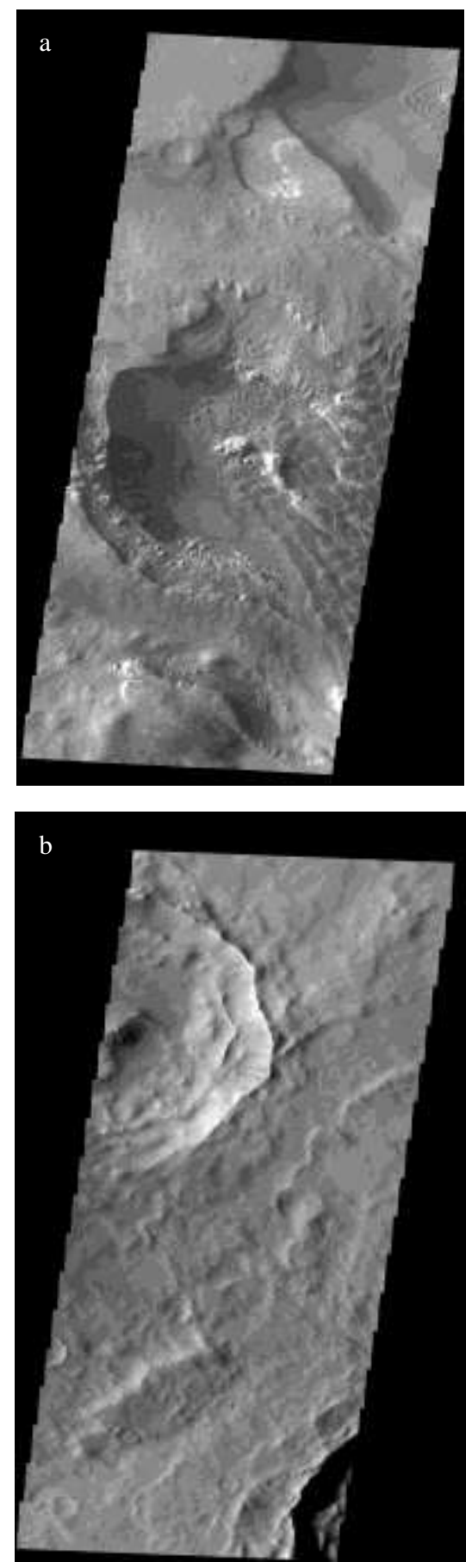

Figure 2. a) Test case 1 b) Test case 2 


\section{RESULTS}

The algorithm has been tested on the images shown in Figure. 2(a) and 2(b). Figure 3 shows the results obtained by proposed algorithm for test case 1. Experiments have been performed at $\alpha=0.29, L=9$ for test case 1 and test case 2. Figure. 3(a), 3(b), 3(c), 3(d), 3(e) and 3(f) shows results obtained at different value of relaxation coefficient $\lambda=0.2,0.7,0.9,1.3,1.7$ and 1.9 . It has been found that for lower value of relaxation coefficient we are able to extract edges accurately.

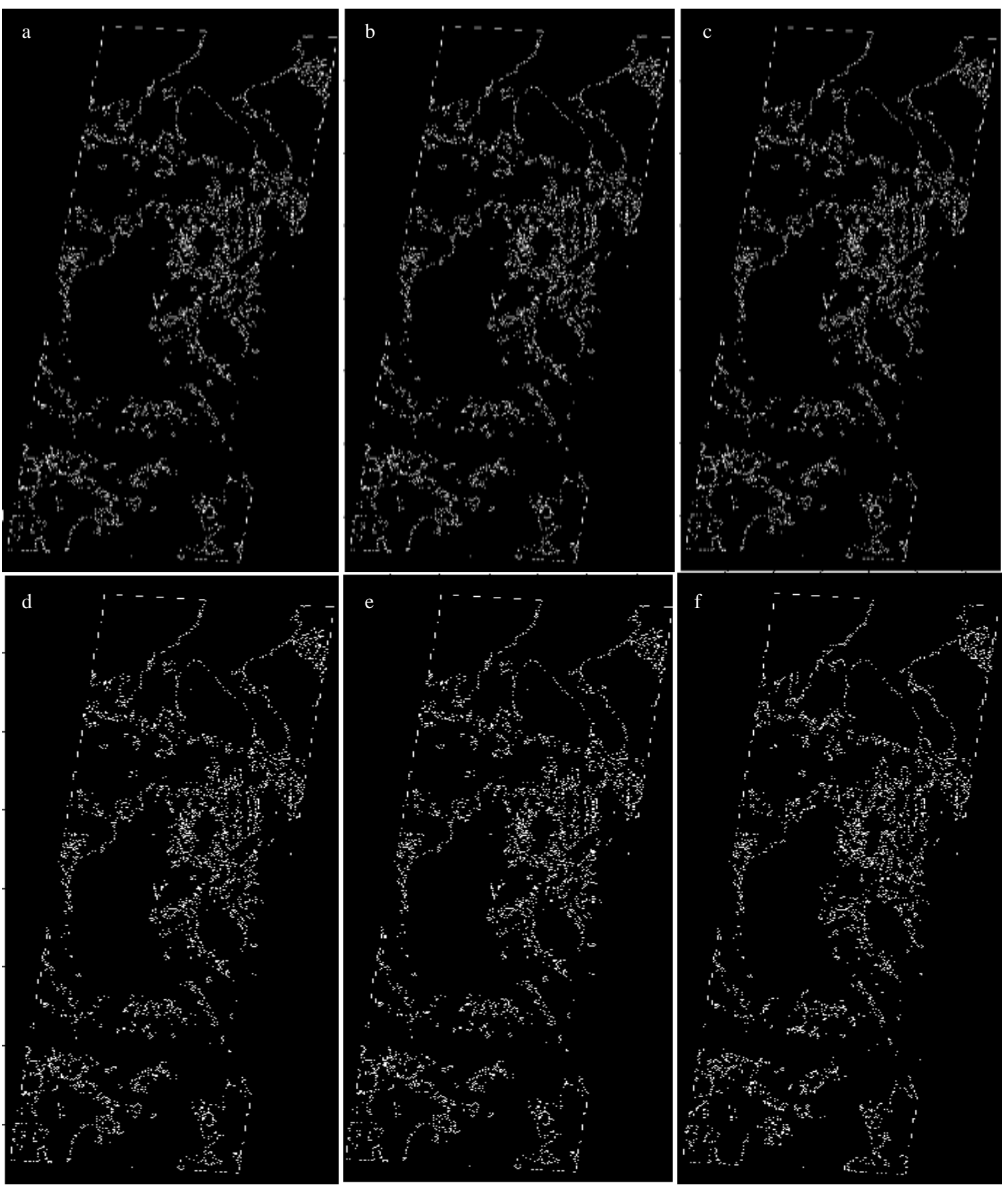

Figure 3. Edges for test case 1 by proposed algorithm a) $\lambda=0.2$ b) $\lambda=0.7$ c) $\lambda=0.9$ d) $\lambda=1.3$ e) $\lambda=1.7$ f) $\lambda=1.9$

Results obtained by proposed algorithm for test case 2 have been shown in Figure. 4. Order of differentiation and filter length for our experiment is 0.29 and 9 , respectively. The Algorithm has been validated for six different value of relaxation coefficient. The value of coefficients is $\lambda=0.2,0.7,0.9,1.3,1.7$ and 1.9 . It clearly shows the edges are clearer for lower value of relaxation coefficient. Figure 5 shows the results obtained for test case 1 by existing edge detection method. Figure 5 (a), 5 (b) and 5 (c) show output images of Prewitt operator, Sobel operator, Roberts operator, 

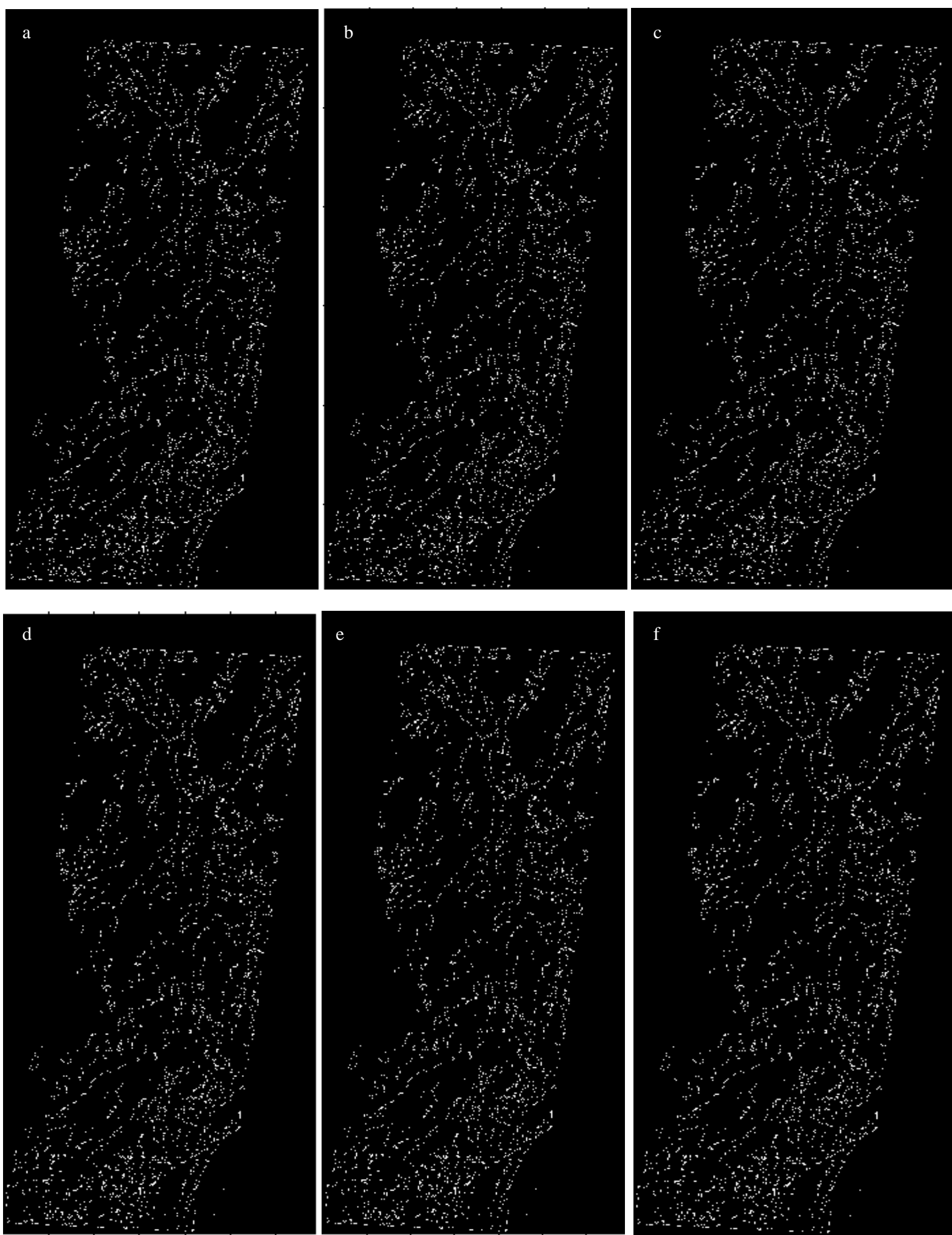

Figure 4 . Edges for test case 2 by proposed algorithm at a) $\lambda=0.2$ b) $\lambda=0.7$ c) $\lambda=0.9$ d) $\lambda=1.3$ e) $\lambda=1.7$ f) $\lambda=1.9$ 

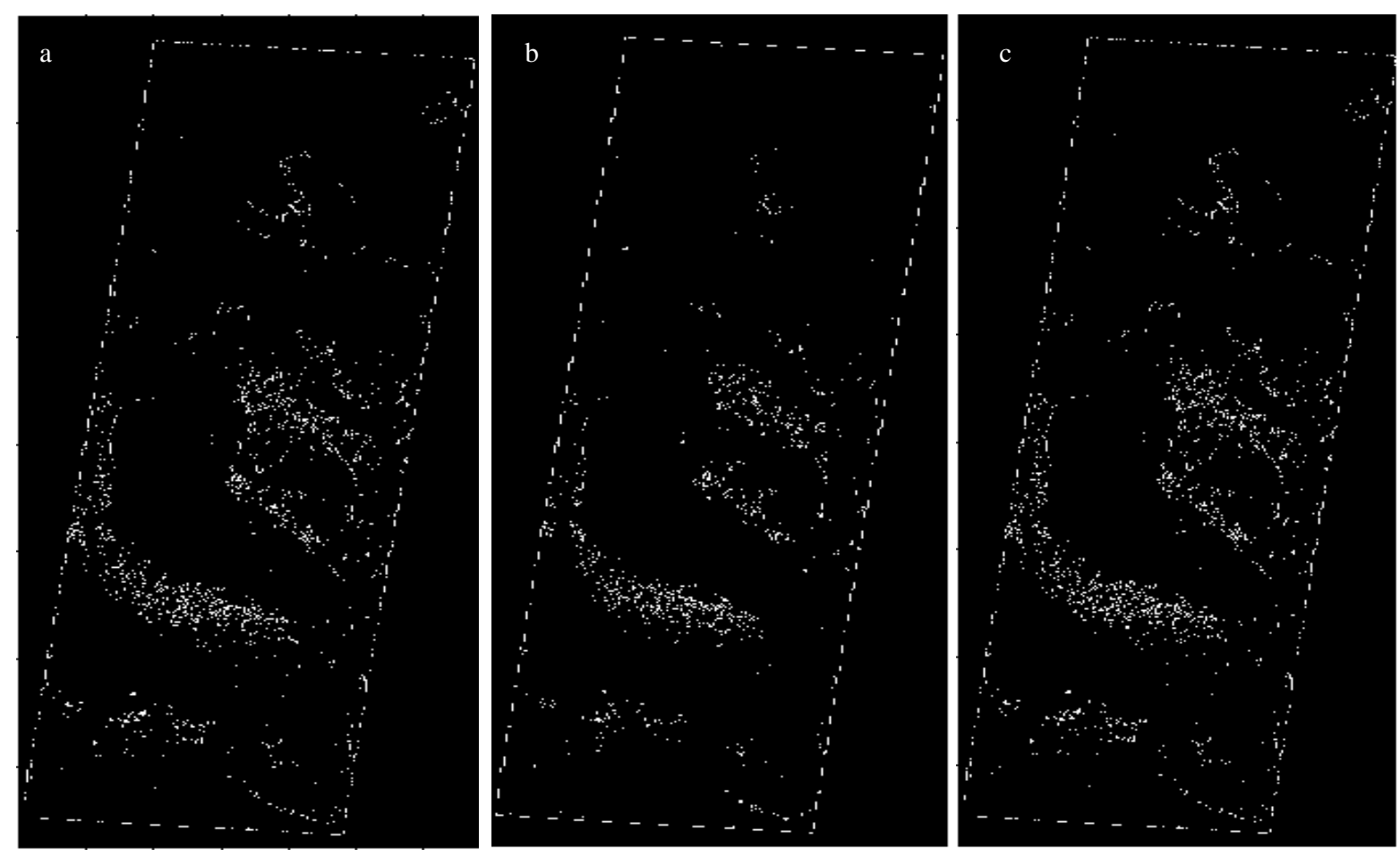

Figure 5. Edges for test case 1 by a) Prewitt method b) Sobel method c) Roberts method
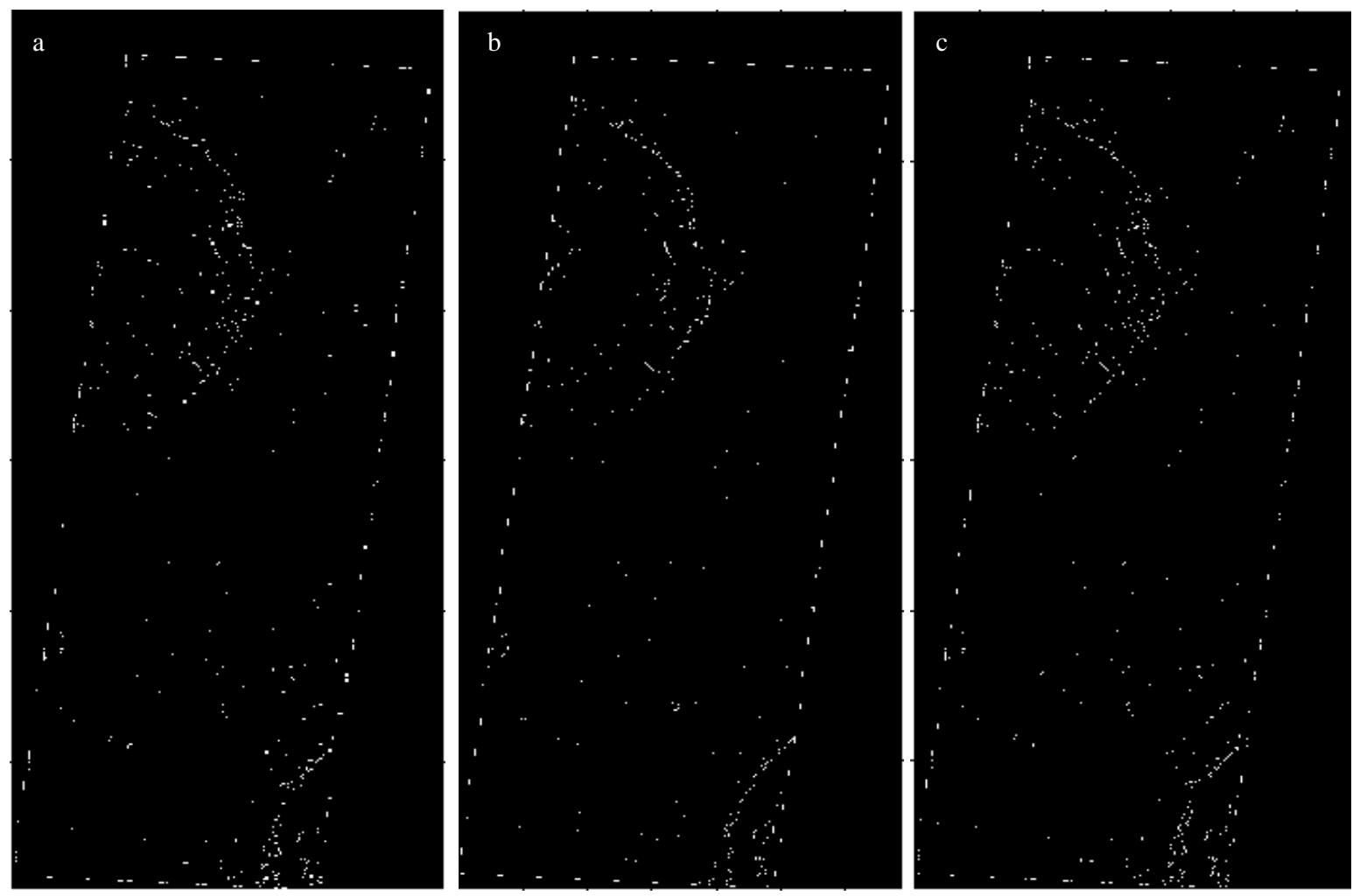

Figure 6. Edges for test case 2 by a) Sobel method b) Roberts method c) Prewitt method

Figure 6 shows the result obtained from the existing algorithms for the test case 2. Figure 6 (a), 6 (b) and 6 (c) are the results obtained from Sobel method, Roberts method and Prewitt method respectively. Result of these methods clearly shows their inefficiency for detecting edges in test case 2 . 


\section{CONCLUSIONS}

It has been found that extracting edges in Satellite images having low contrast and smoothness properties is very difficult. The traditional masking based edge detection algorithms are not giving good results for these types of images. Design of new filter has play major part in proposed algorithm. The preprocessed input image followed by Sobel edge detector has been performing well. It has been observed that algorithm is performing well for lower values of relaxation coefficient.

\section{REFERENCES}

Canny, J., 1986. A Computational Approach to Edge Detection, IEEE Trans. Pattern Analysis and Machine Intelligence, 8 (6), pp. 679- 698.

Chen, D., Chen,Y.Q., Xue, D., 2011. Digital fractional order SavitzkyGolay differentiator, IEEE Trans. on Circuits and Systems-II: Express Briefs, 58, pp. 758-762.

Deng, G., and Pinoli, J.C., 1998. Differentiation-based edge detection using the logarithmic image processing model, J. Math. Imaging vision 8 pp. $161-180$.

Deriche, R., 1990. Fast algorithms for low-level vision, IEEE Trans, PAMI, Vol.12 no A pp. 78-87.

Gonzalez, R.C. and Woods, R. E., 2009. Digital Image Processing, Third Edition, Pearson Education, New Delhi.

Guillon, S., Baylon, P., Najim, M. and Keskes, N., 1998. Adaptive nonlinear filters for 2-D and 3-D image enhancement, Signal Processing, Vol. 67, pp.237-254.

Hou, Jie, Ye, Jin-Hua, and Sha-Sha, Li, 2007. Application of canny combining and wavelet transform in the bound of step-structure edge detection", 2007 International Conference on Wavelet Analysis and Pattern Recognition, IEEE, USA, pp.1635-1637.

http://photojournal.jpl.nasa.gov/catalog/PIA10300.

Iyengar, S.S. and Deng, W., 1995. An efficient edge detection algorithm using relaxation labeling technique, Pattern Recognition, 28 pp. 519-536.

Johnson, R.P., 1990. Contrast based edge detection, Pattern Recognition, 23, pp. 311-318.

Man, D., 1982.Vision. San Francisco, CA: Freeman.

Marrand, D. and Hildreth, E., 1980. Theory of Edge Detection, Proc. Roy. Soc. London, vol. B207, pp. 187-217.

Mitra, S.K., 2004. Digital Signal Processing: A computer based approach, McGraw-Hill India edition.

Oldham, K.B. and Spanier, J., 1974. Eds., The Fractional Calculus. New York: Academic.

Pandey R.K., Suman S., Singh Koushlendra K. and Singh O.P., 2014. An Approximate Method for Abel Inversion using Chebyshev Polynomials, Applied Mathematics and Computation, vol. 237, pp.120132.

Perona, P., and Malik,J., 1990. Scale-space and edge detection using anisotropic diffusion, IEEE Trans. Pattern Anal. Mach. Intell. Vol. 12 pp. 629-639.
Prewitt, J.M.S., 1970. Object Enhancement and Extraction Picture Processing and Psychopictorics, B. Lipkin and A. Rosenfeld, eds., New York: Academic, pp. 75-149.

Podulubny, I., 1998. Fractional Differential Equation, Mathematics in Science \& Engineering, Academic Press: California, USA.

Rivlin,T.J., 1974. The Chebyshev polynomials, John Wiley \& Sons press.

Rosenfeld, A., and Thurston, M., 1971. Edge and curve detection for visual scene analysis, IEEE Trans. Cornput., vol. C-20, pp. 562-569.

Shen, J, and Castan, S., 1992. An Optimal Linear Operator for Step Edge Detection Computer Vision Graphics Image Processing, vol. 54 no. 2 , pp. $112-13$

Singh, K. K, Bajpai, M., K., and Pandey, R., K., 2014. Report on "A Novel Approach for Enhancement of Geometric and Contrast Resolution Properties of Low Contrast Images", IIITDMJ/CSE/2014/PGR0108.

Witkin, A. P., 1983. Scale-space filtering in Proc. IJCAI-8.

Yuille, A. L, and Poggio,T., 1986. Scaling theorems for zero-crossings IEEE Trans. Pattern Anal. Machine Intell., PAMI-8, pp. 15-25. 